Int. J. Morphol.,

35(1):331-336, 2017

\title{
Cambios Morfológicos en la Ontogenia Temprana de Chirostoma riojai
}

\author{
Morphological Changes in the Early Ontogeny of Chirostoma riojai
}

Iván Alarcón-D. ${ }^{\text {; }}$ Manuel Arnoldo Castillo-Rivera² ${ }^{2}$ Joaquin Arroyo-Cabrales $^{3}$ \& Gerardo Figueroa-Lucero

\begin{abstract}
ALARCÓN-D. I.; CASTILLO-RIVERA, M. A.; ARROYO-CABRALES, J. \& FIGUEROA-LUCERO, G. Cambios morfológicos
\end{abstract} en la ontogenia temprana de Chirostoma riojai. Int. J. Morphol., 35(1):331-336, 2017.

RESUMEN: El charal de Toluca Chirostoma riojai es un pez de alto valor cultural e ictiológico. Los trabajos sobre esta especie se enfocan en el tipo de alimentación o forman parte de revisiones taxonómicas y son pocos los estudios sobre los cambios morfológicos durante su ontogenia. Se evaluó la figura de C. riojai mediante morfometría geométrica desde su eclosión hasta los 90 días de vida. Los ejemplares se cultivaron en condiciones controladas y fueron fijados 171 organismos pertenecientes a 11 intervalos de edad incluidos en cinco fases: embrión libre, apterolarva, pterolarva, juvenil y adulto. Se usaron seis puntos anatómicamente homólogos en una primera configuración de marcas y en una segunda se delinearon dos contornos (dorsal y ventralmente) de la región anterior. Un análisis de componentes principales mostró una fuerte diferenciación en su figura durante la transición larva-juvenil, en tanto un análisis de variables canónicas señaló diferencias significativas (ps<0.001) entre los 11 grupos de edad. La regresión lineal entre el logaritmo del tamaño centroide y las distancias procustes muestran que los organismos mantienen un cambio de figura constante hasta el término del periodo larvario, punto en el que el periodo juvenil comienza y las modificaciones en su figura disminuyen. La descripción del desarrollo temprano de $C$. riojai sin considerar el desarrollo de las aletas, muestra que la característica con el mayor cambio es el desplazamiento del poro anal, seguido por el alargamiento de la región cefálica. El tamaño y la figura de $C$. riojai durante su vida temprana pueden servir para diferenciar faces de desarrollo y ser utilizada en otras especies hermanas para evaluar si su figura es específica en cada fase. Los cambios morfológicos de C. riojai durante su vida temprana se pueden describir de forma gradual hasta el periodo larval y saltatoria en el cambio a juvenil.

PALABRAS CLAVE: Morfometría geométrica; Figura; Ontogenia temprana; Chirostoma.

\section{INTRODUCCIÓN}

El charal de Santiago, Chirostoma riojai Solórzano y López, 1966 (Osteichthyes: Atherinopsidae), se distribuye en embalses de la cuenca del alto Lerma, México; actualmente se localiza solo en cuatro embalses pertenecientes al Alto Lerma, todos en el Estado de México (Figueroa et al., 2003). Esta especie es zooplanctófaga, aunque se ha reportado el consumo de larvas y adultos de insectos, así como peces de su misma especie (Vilchis, 2000).

Es evidente que los cambios durante la historia de vida temprana en los peces incluye una serie de cambios morfológicos, fisiológicos y ecológicos (Yauson, 1988; Balon, 2001), los cuales denotan etapas, períodos o fases de desarrollo según sea el autor que se consulte (Helfman et al., 2009). La ontogenia en los peces es considerada como un proceso plástico que es susceptible a los cambios en las condiciones (Kouttouki et al., 2006). Es por esto, que los cambios morfológicos que sufren durante su ontogenia están estrechamente relacionados con su hábitat (Macpherson, 1998). Una de las condiciones ambientales que afecta o determina los tiempos de desarrollo y aspectos fisiológicos es la temperatura (Angilleta et al., 2004), ya que esta puede modificar las tasas de crecimiento e influye en la determinación del sexo de estos organismos (Ito et al., 2005; Strussmann et al., 2010).

Los trabajos de descripción morfológica posterior a la fase de embrión libre para el género Chirostoma son pocos y se basan principalmente en determinar la supervivencia y tasa de crecimiento de los ejemplares, describiendo únicamente la morfología basada en la aparición de las aletas y otras estructuras como la placa hipúrica. En los estudios que se describen el crecimiento y el desarrollo temprano de los peces se utiliza la morfometría tradicional como herramienta para su análisis. Así en general, los pocos estudios de C. riojai y de otras especies del género se han des-

\footnotetext{
${ }^{1}$ Maestro en Biociencias, Doctorado en Ciencias Biológicas y de la Salud, UAM-I. Ciudad de México, México.

${ }^{2}$ Doctor en Ciencias, Universidad autónoma metropolitana, Ciudad de México, México.

${ }^{3}$ Doctor en Ciencias, Profesor-Investigador SSLA-INAH, Ciudad de México, México.
} 
crito con morfometría lineal (Figueroa-Lucero et al., 2004; Hernández-Rubio \& Lucero, 2013).

Recientemente el uso de la Morfometría Geométrica (MG) ha permitido que la descripción de la figura de los peces durante su desarrollo sea con mayor precisión, además da la posibilidad de realizar análisis multivariados robustos (Roholf, 1998).

El estudio morfológico de C. riojai es importante para entender las dinámicas poblacionales y poder examinar que condiciones pueden afectar su desarrollo en las diferentes fases de su historia de vida, especialmente porque esta es una especie de interés comercial, cultural y de alto valor ictiológico. Así, el objetivo del presente trabajo fue describir el cambio de figura de $C$. riojai durante el desarrollo temprano hasta el principio del periodo adulto.

\section{MATERIAL Y MÉTODO}

Ejemplares. Se analizaron 171 ejemplares entre 0 a 90 días después de la eclosión (DDE) considerando once intervalos de edad (Tabla I). A partir de adultos de la población de $C$. riojai de la Presa San Jacinto (19 $39^{\prime}$ 'N y $99^{\circ} 45^{\prime}$ W) Estado de México, se obtuvieron los óvulos que se fertilizaron in vitro y se mantuvieron en condiciones constantes de luz (12:12), temperatura $\left(20 \pm 2{ }^{\circ} \mathrm{C}\right)$ y salinidad $(0 \mathrm{~g} \mathrm{NaCl} / \mathrm{l})$, hasta su eclosión en el décimo día. Las cohortes se cultivaron bajo condiciones controladas de luz y temperatura $\left(12: 12\right.$ y $\left.20 \pm 2{ }^{\circ} \mathrm{C}\right)$, con suministró de oxigeno $(5,4 \mathrm{mg} / \mathrm{L})$. A partir del segundo día después de la eclosión, se suministró rotíferos Brachionus plicatilis a los embriones libres hasta la fase de apterolarva. Posteriormente se proporcionaron nauplios de Artemia sp. y alimento balanceado para trucha durante la fase de Pterolarva hasta alcanzar el período juvenil, en la que además se les suministró pulga de agua Daphnia magna.

Morfometría Geométrica (MG). Se tomaron fotografías digitales del lado izquierdo del pez, con una cámara CANON RebelT3i (16 mpx) montada sobre un microscopio estereoscópico Olympus. Se colocaron seis marcas anatómicas y homólogas de referencia (landmarks) a lo largo del cuerpo, las cuales corresponden a puntos homólogos y constantes durante todo su desarrollo (del grupo uno al once); configuración que se basó en la propuesta por Kouttouki et al. (2006) (Figs. 1 A y B). Adicionalmente se evaluaron partir del embrión libre hasta el comienzo del periodo juvenil (del grupo uno al siete) los contornos dorsal y ventral de la región anterior del pez utilizando un total de 18 puntos (Figs. $1 \mathrm{C}$ y D). Para los análisis de MG se usó el programa TPSdig (Zelditch et al., 2004) y el programa Makfan para poner las marcas de los contornos. Las coordenadas se alinearon usando el método generalizado de superposición de procrustes por mínimos cuadrados, procedimiento mediante el cual se elimina la información de tamaño, posición y rotación de las coordenadas (Rohlf \& Slice, 1990). Los Partial Warps se obtuvieron con los programas de la serie Integrate Morphometrics Package (IMP) (Sheets, 2004), con los cuales se uniformiza la figura de los organismos para describir las diferencias en la figura durante la ontogenia temprana (Zelditch et al.).

Análisis multivariados. Para hacer una descripción del cambio de la figura se usó un Análisis de Componentes Principales (ACP), análisis del cual también se obtuvo la información para crear las mallas de deformación. La significación en las diferencias en la figura de cada grupo de edad se evaluó con un Análisis de Variables Canónicas (AVC). Por último, se realizó una regresión lineal entre el Logaritmo del Tamaño
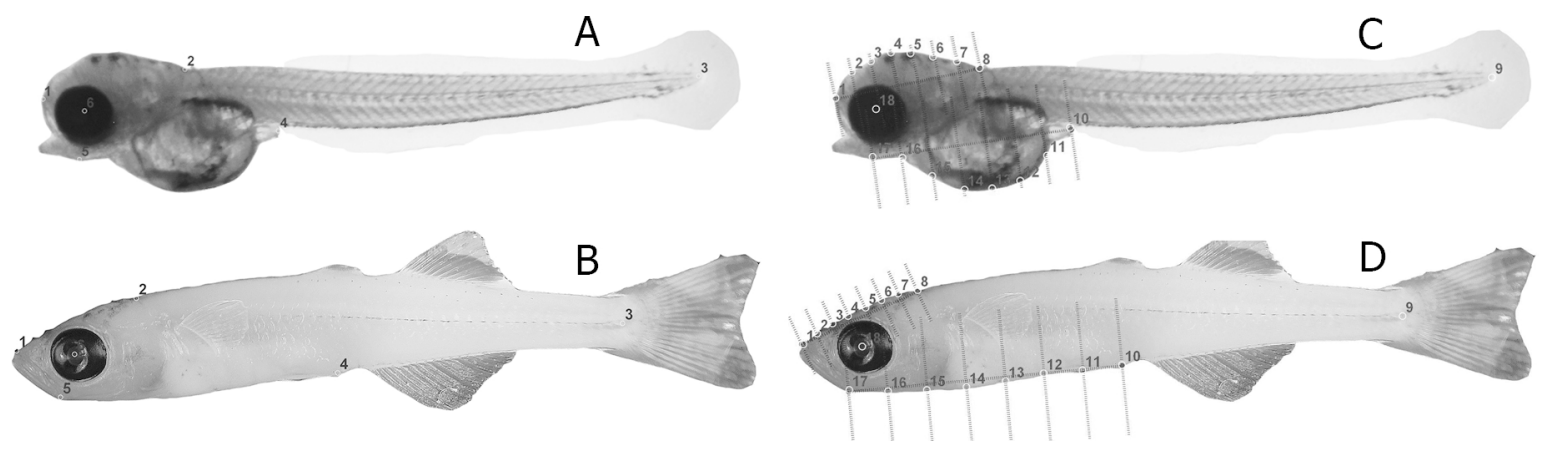

Fig. 1. Posición de las marcas en C. riojai: A-B (embrión libre y juvenil respectivamente):1-inicio del premaxilar, 2-fin de la región cefálica, 3-región media de la aleta caudal en donde termina la notocorda, 4-orificio anal, 5-región central del ojo y 6-región anterior de la mandíbula. C-D (embrión libre y juvenil): 1-(la región distal del premaxilar) a la 8 (el término de la región cefálica) son la base del primer peine usado para delinear la región dorsal de la parte anterior del pez; 9 región media de la aleta caudal en donde termina la notocorda ; 10-(la apertura anal) a la 17 (articulación de la mandíbula ) son la base del segundo peine usado para delinear la región anterior en vista ventral y 18 región central del ojo. 
Tabla I. Edades en días después de la eclosión (DDE) e intervalos de los mismos.

\begin{tabular}{|c|c|c|c|c|}
\hline $\mathrm{DDE}$ & $\begin{array}{l}\text { Grupo } \\
\text { de edad }\end{array}$ & Fase & Descripción & Símbolo \\
\hline 0 a 2 & 1 & $\begin{array}{l}\text { Embrión } \\
\text { libre }\end{array}$ & $\begin{array}{l}\text { Presenta una aleta embrionaria, el saco vitelino y la alimentación es } \\
\text { endógena (Balon, 1981). }\end{array}$ & \\
\hline 3 a 10 & 2 & Apterolarva & \multirow{2}{*}{$\begin{array}{l}\text { Comienza con la alimentación mixta, después exógena y termina con el } \\
\text { comienzo de la flexión de la notocorda (Balon, 1981). }\end{array}$} & \\
\hline 11 a 20 & 3 & Apterolarva & & \\
\hline 21 a 30 & 4 & Pterolarva & \multirow{3}{*}{$\begin{array}{l}\text { Inicia con el desarrollo de la placa hipúrica y con la aparición de las } \\
\text { aletas, (Balon, 1981). }\end{array}$} & \\
\hline 31 a 40 & 5 & Pterolarva & & \\
\hline 40 a 48 & 6 & Pterolarva & & \\
\hline 49 a 57 & 7 & Juvenil & \multirow{4}{*}{$\begin{array}{l}\text { El desarrollo de las aletas ha concluido, se da la formación de las } \\
\text { escamas (Balon, 1981). }\end{array}$} & \\
\hline 57 a 65 & 8 & Juvenil & & \\
\hline 65 a 73 & 9 & Juvenil & & \\
\hline 74 a 81 & 10 & Juvenil & & \\
\hline 81 a 90 & 11 & Adulto & Maduración gonádica (Balon, 1981). & \\
\hline
\end{tabular}

Centroide (LogTc) y las distancias geométricas para evaluar la relación entre la figura y el tamaño centroide. Se utilizaron los programas PCAGen, CVAGen y Regreess de la serie IMP (Sheets) para estos análisis multivariados. El programa PAST (Hammer et al., 2001) se utilizó en la edición de los gráficos.

\section{RESULTADOS}

Los once intervalos de edad se encuentran clasificados en las fases de embrión libre, apterolarva, pterolarva, juvenil y adulto (Tabla I). De acuerdo con los resultados del ACP, los organismos se ordenan acorde al intervalo de edad que van del uno al once en las dos primeras componentes, con una varianza acumulada del $92,11 \%$ (Fig. 2). Los organismos a partir del embrión libre hasta el periodo larvario (edades uno-seis) se distribuyen del lado negativo del CP1 separándose claramente de los juveniles y adultos (edades siete-once), los cuales se ubican del lado positivo del mismo componente.
La malla de deformación obtenida a partir del ACP se detalla como la posición del ano se dirige hacia la parte posterior del cuerpo conforme avanza en su ontogenia y es uno de los cambios más significativos durante su desarrollo (Fig. 3 A),

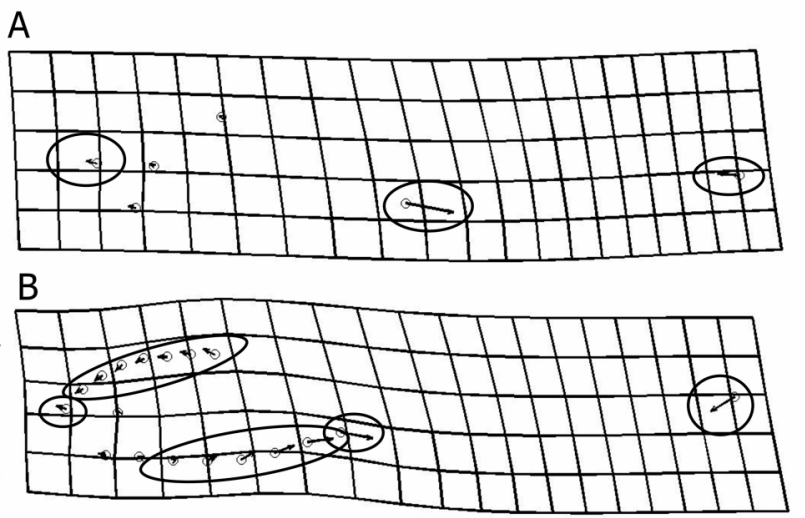

Fig. 3. Mallas de deformación de C. riojai a partir del primer componente (CP1) del ACP.: A. análisis de la figura solo con marcas, B Análisis de la figura con marcas y contornos, resaltando las marcas con mayor cambio.
Fig. 2. Análisis de Componentes Principales aplicado a todos los individuos de los 11 grupos de edad de $C$. riojai. El símbolo de cada grupo de edad de acuerdo con la Tabla I. Se observa una disposición en orden progresivo, del lado izquierdo los que corresponden de embrión libre hasta el final del periodo larvario y del lado derecho se distribuyen solo los juveniles y los primeros organismos adultos.

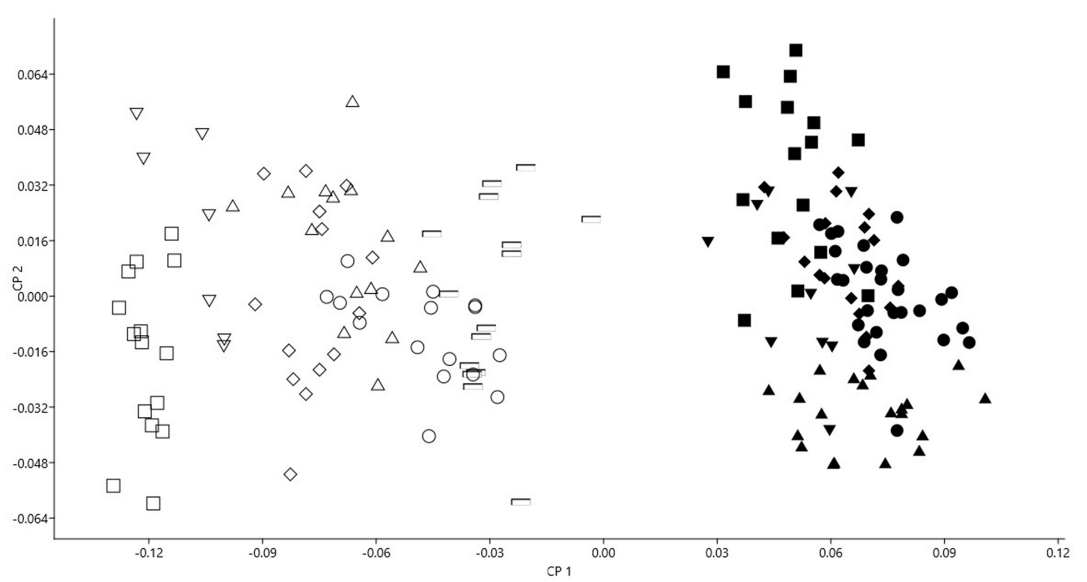


mientras que un cambio menos conspicuo se observa en la región caudal, así como en el premaxilar (las cuales van en dirección a la región anterior). Asimismo, los resultados de la región cefálica (Fig. 3 B) muestran que su contorno se dirige hacia la región anterior y por el contrario, el contorno que está sobre el saco del vitelo se dirige hacia la parte posterior de los organismos.

Los resultados del AVC señalaron diferencias significativas entre los once grupos de edad (para las dos prime- ras funciones $\lambda$ 's $<0,06$, p's $<0,001$ y una varianza acumulada de $95,45 \%$ ). En la relación de la figura con el logaritmo del tamaño centroide (Fig. 4), aunque algunos de los organismos parecen que se mezclan tanto por su tamaño como por su figura con los de otras edades, se observa un claro patrón de separación a partir del sexto grupo de edad, lo que se ve reflejado en el tamaño centroide. También se observa un cambio en el comportamiento de la figura, el cual es continuo hasta el sexto grupo de edad, pero a partir del grupo siete, la figura se mantiene relativamente constante con una dispersión más homogénea de los organismos.

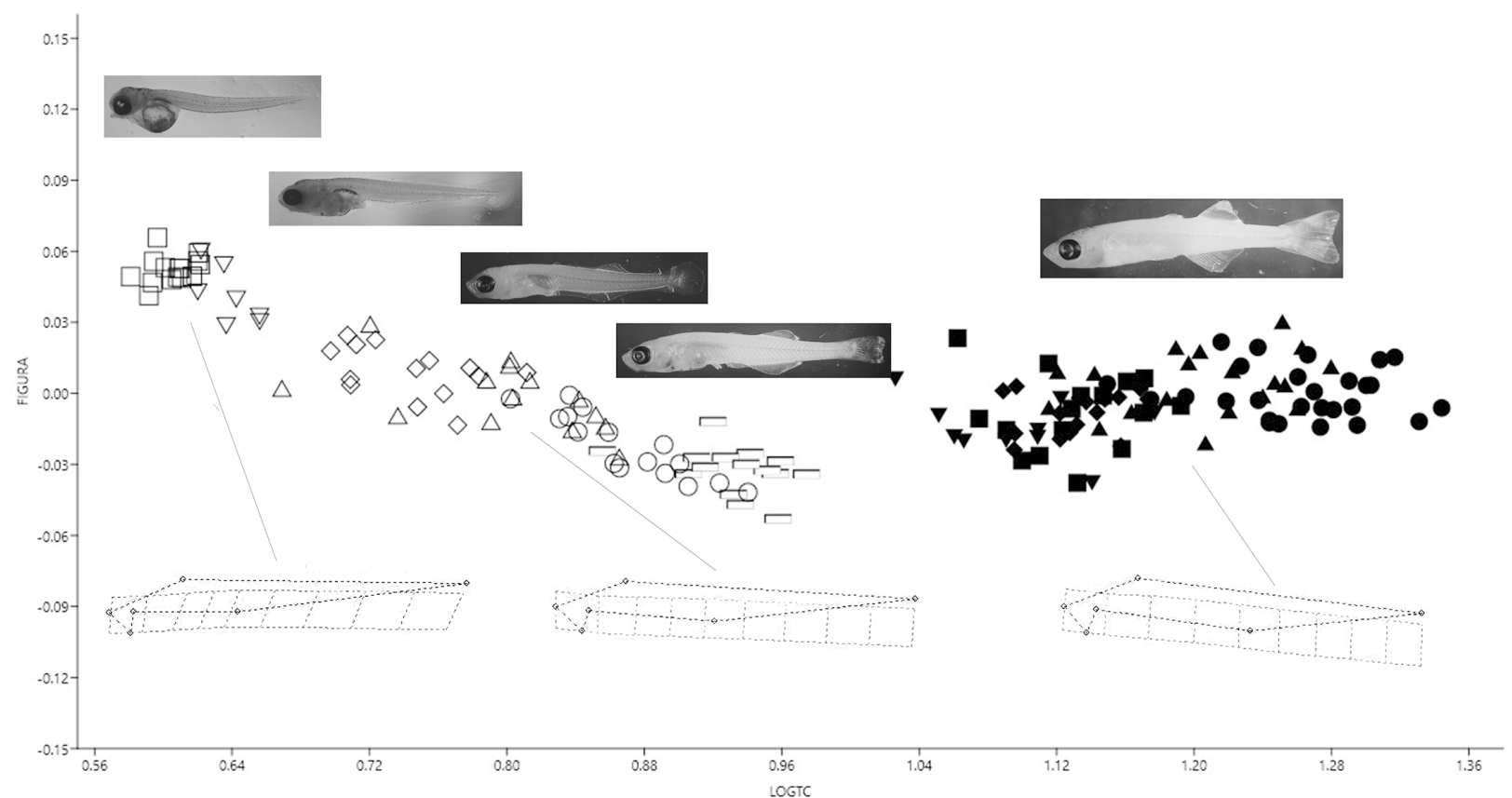

Fig. 4. Dispersión de los organismos de C. riojai considerando la figura y el logaritmo del tamaño centroide (LOGTC), mostrando solo las mallas de deformación del embrión libre, la larva y el juvenil, sí como imágenes del embrión libre, la apterolarva, pterolarva inicial - final y al último el juvenil en orden ontogenético

\section{DISCUSIÓN}

El ciclo de vida en los peces se explica con base en sus características fisiológicas o morfológicas dado que se presentan cambios que describen pasos, fases, etapas o periodos de desarrollo. En general los períodos de embrión, larva, juvenil y adulto pueden englobar cada aspecto de su ontogenia. Sin embargo, existen enfoques distintos en la terminología utilizada y teorías opuestas acerca de si es un desarrollo gradual como comúnmente se ha manejado o por un desarrollo saltatorio (Greenwood, 1989; Balon; Helfman et al., 2009).

Los peces al momento de la eclosión presentan una figura inicial básica, que se caracteriza por la presencia de una aleta embrionaria continua que inicia donde termina la región cefálica en la parte dorsal y concluye en el poro anal, posteriormente dicha aleta se reabsorbe y comienza el desarrollo del resto de las aletas. Existen algunas etapas del desarrollo como el término de la apterolarva y el inicio de la pterolarva que no tienen una clara separación en su figura o no es tan evidente, aunque estas dos etapas se pueden diferenciar por características como la aparición de radios en la aleta dorsal o la flexión de la notocorda (Balon); en tanto su figura es difícil de acotar si no se consideran estas características. Si se entiende que el estudio de la forma sensu stricto mediante la MG distingue entre datos de "Forma" (Form) que se obtienen al eliminar los efectos de rotación y traslación, de los datos de "Forma Pura" (Shape) o "Figura" en donde además se contempla la eliminación del efecto del tamaño, se puede obtener figuras reales de cada una de las pasos, etapas, fases o periodos durante la ontogenia. 
En este sentido, el desarrollo de estructuras como los radios, en un principio no influyen drásticamente en el cambio de figura del pez, pero sí son características importantes en la separación de algunas de las fases dentro del periodo larvario (Balon), por lo que se puede decir que los cambios morfológicos son continuos en las etapas tempranas de desarrollo y dichos cambios disminuyen después del periodo de juvenil en donde ya no se forman o desarrollan más estructuras, a diferencia de los cambios fisiológicos que presentan cambios cuando pasan de una fase o periodo a otro (Balon).

Cabe hacer mención, que al inicio del desarrollo de una estructura como una aleta, no la hace una estructura funcional; hasta que la aleta está completamente formada se puede decir que su funcionalidad es óptima o es adecuada hasta que está completamente desarrollada (Weibel et al., 1991). Independientemente de las estructuras que crecen durante el desarrollo, la figura de $C$. riojai es en general fusiforme durante casi toda su ontogenia, a diferencia de otros peces donde su figura cambia drásticamente de ser fusiforme a algo parecido a un rombo comprimido al llegar a ser juvenil (Loy et al., 1998).

Bajo la teoría saltatoria propuesta por Balon, Hernandez-Rubio et al. (2006) se encontró que en el desarrollo temprano de $C$. humboldtianum la tasa de crecimiento absoluto de la distancia notocordal y la región cefálica de los embriones libres es mínima ya que se da prioridad al desarrollo de órganos y sistemas, dando lugar a un umbral en donde después se da paso al crecimiento, aspectos que si bien permiten definir fases, no describe los cambios de figura durante su crecimiento. La teoría saltatoria menciona que existen umbrales (en general asociados a aspectos fisiológicos) entre las diferentes fases de desarrollo, para lo cual es necesario tener ciertas características antes de pasar de una etapa a otra (Balon). La figura de $C$. riojai muestra cambios continuos desde la eclosión del embrión hasta el periodo larvario (Fig. 4), después del cual hay un fuerte cambio al paso juvenil, siendo evidente una estabilidad en los periodos de juvenil y adulto. Esto se debe a que terminó el desarrollo de estructuras y sistemas, enfocando su energía solo en el crecimiento (Fig. 4).

La figura de $C$. riojai durante la etapa de embrión libre cambia después de que el saco vitelino es consumido, por tanto la figura redondeada en la región ventral pasa a ser casi horizontal al inicio de la apterolarva. Posterior a la fase de embrión libre, algunos de los organismos pueden tener tallas mayores o menores que la media de su correspondiente cohorte de edad, por lo que su figura se asemeja más a los ejemplares de su misma talla.

Durante el periodo larval entre los 10 y los 30 DDE los cambios de figura también son notorios no solo en la figu- ra evaluada, también por el desarrollo de las aletas dorsales, pélvicas y anal, características que le permite al organismo tener una capacidad mayor de movimiento, conjuntamente con la modificación de la mandíbula y con ello un mejor aprovechamiento de los recursos (Chalde et al., 2011).

Es claro que al término del periodo larvario y en el comienzo del periodo de juvenil existe un gran cambio en la figura y posteriormente las diferencias de figura son menores debido a que se encuentra en una aparente etapa de estabilidad (Loy et al., 2001), puesto que el desarrollo de estructuras externas e internas fue concluido y solo falta que sus gónadas maduren para alcanzar el periodo de adulto los organismos se dedican a incrementar su tamaño (Balon).

Así como en otros aterinópsidos (Chalde et al.), es evidente que los tiempos de DDE para que cada periodo o fase en cada organismo son diferentes y cada cohorte tiende a tener intervalos que pueden variar hasta en más de siete días, es decir, en una misma cohorte de peces existen organismos con diferente grado de desarrollo y el tamaño del centroide puede diferenciar dichas fases o etapas y sus correspondientes tiempos de DDE. No obstante lo anterior, es claro que la mayoría de los organismos mantienen una sincronía durante su ontogenia, lo que permite estimar cuando una cohorte alcanza una fase de desarrollo y cuando la termina.

El cambio en la posición del ano y el alargamiento de la región cefálica son las dos características de la figura que sufren una mayor modificación durante la ontogenia temprana después del desarrollo de las aletas, que en conjunto dan la forma general a la especie. Es por esto que el conocer cómo se dan los cambios durante el desarrollo temprano, permite hacer asociaciones entre la figura de las especies, las condiciones y los recursos que necesitan para poder implementarlos con propósitos de producción acuícola (Kouttouki et al.; Asgari et al., 2013). El tamaño y figura de los organismos pueden ser un indicador de la fase de desarrollo en la que se encuentran, aspectos morfológicos distintos a los convencionales y más fáciles de observar que los cambios fisiológicos, por lo que esta información puede ser utilizada en la separación de etapas tempranas de las diferentes especies del género Chirostoma que se encuentran en simpatría.

AGRADECIMIENTOS. El presente trabajo forma parte del proyecto doctoral de Iván Alarcón Durán perteneciente al Doctorado en Ciencias Biológicas y de la Salud de la Universidad Autónoma MetropolitanaIztapalapa Ciudad de México, México, contando con Beca CONACYT 359096. Se agradece al laboratorio de Biología del Desarrollo del departamento de Hidrobiología UAM-I por proporcionar las instalaciones para el cultivo de los ejemplares para la realización de este estudio. Asimismo, al Laboratorio de Arqueozoología "M. en C. Ticúl Álvarez Solorzano" de la SLAA-INAH por permitir usar el equipo necesario y proporcionar el espacio para realizar los análisis de MG. 
ALARCÓN-D. I.; CASTILLO-RIVERA, M. A.; ARROYOCABRALES, J. \& FIGUEROA-LUCERO, G. Morphological changes in the early ontogeny of Chirostoma riojai. Int. J. Morphol., 35(1):331$336,2017$.

SUMMARY: Toluca silverside Chirostoma riojai is a fish with high cultural and ictiological values. Studies on this species have focused on the type of diet or as part of taxonomic reviews, while few reports are known on the morphological changes during its ontogeny. In this study the shape of $C$. riojai was evaluated by means of geometric morphometrics from hatchings up to 90 days-old individuals. Specimens of $C$. riojai were cultivated in controlled conditions, and 171 organisms there fixed, belonging to 11 age intervals included within five phases: free embryo, apterolarva, pterolarva, juvenile, and adult. There were used six landmarks in an initial configuration, and in a second configuration there were outlined two contours (dorsal and ventral) on the anterior region. A principal component analysis showed a strong differentiation in the shape during the transition larva-juvenile, for both an analysis of canonical variables found significant differences (ps<0.001) between the 11 age groups. The linear regression between the logarithm of the size centroid and procustes distances, showed that the individuals maintain a constant change in shape up to the end of the larval phase, at which time the youth stage begins and the changes in its shape decreases. The description of the early development of $C$. riojai without considering the development of the fins, shows that feature with greatest change is the movement of the anal pore, followed by the lengthening of the cephalic region. The size and shape of $C$. riojai during its early life could serve to differentiate developmental phases and could be used with other sister species to assess whether their shape is specific in each phase. The morphological changes of $C$. riojai during his early life could be described in a gradual form up to the the larval period, while saltatory in the change to juvenile.

KEY WORDS: Geometric morphometrics, shape, early ontogy, Chirostoma.

\section{REFERENCIAS BIBLIOGRÁFICAS}

Angilletta, M. J. Jr.; Steury, T. D. \& Sears, M. W. Temperature, growth rate, and body size in ectotherms: fitting pieces of a life-history puzzle. Integr. Comp. Biol., 44(6):498-509, 2004.

Asgari, R.; Eagderi, S.; Rafiee, G.; Poorbagher, H.; Agh, N. \& Zadeh, H. E. Body shape changes during the early development of the Beluga (Huso huso). Int. J. Aquat. Biol., 1(1):1-5, 2013.

Balon, E. K. Saltatory ontogeny and the life-history model: neglected processes and patterns of evolution. J. Bioecon., 3(1):1-26, 2001

Chalde, T.; Fernández, D. A.; Cussac, V. E. \& Somoza, G. M. The effect of rearing temperature in larval development of pejerrey, Odontesthes bonariensis: morphological indicators of development. Neotrop. Ichthyol., 9(4):747-56, 2011.

Figueroa-Lucero, G.; Paulo-Maya, J. \& Hernández-Rubio, M. C. Retrospectiva y Avances en el Conocimiento de la Biología y Ecología de los Charales y Peces Blancos del Género Chirostoma (Atheriniformes:Atherinopsidae) en la ENCB-IPN. In: Rojas-Carrillo, P., Fuentes-Castellano, D. (Eds.). Historia y Avances del Cultivo de Pescado Blanco. Ciudad de México, Instituto Nacional de la Pesca, Secretaría de Agricultura, Ganadería, Desarrollo Rural, Pesca y Alimentación, 2003. pp.29-48.

Figueroa-Lucero, G.; Hernández-Rubio, C. M.; Meza, O.; Arredondo-Figueroa, J. L.; Castro, T.; Barriga, I. \& Rodríguez, A. Effect of food type on growth and survival of Chirostoma riojai Solórzano y López, 1965 (Atheriniformes: Atherinopsidae) during early development. J. Biol. Res., 2:93-9, 2004.

Greenwood, P. H. Ontogeny and Evolution: Saltatory or Otherwise? In: Bruton, M. N. (Ed.). Alternative Life-history Styles of Animals. Dordrecht, Kluwer Academic Publishers, 1989.
Hammer, Ø.; Harper, D. A. T. \& Ryan, P. D. PAST: Paleontological Statistics Software Package for Education and Data Analysis. Palaeontol. Electron., 4:1-9, 2001.

Helfman, G. S.; Collette, B. B.; Facey, D. E. \& Bowen, B. W. The Diversity of Fishes: Biology, Evolution, and Ecology. $2^{\mathrm{da}}$ ed. Hoboken, Wiley-Blackwell, 2009.

Hernández-Rubio, M. C. \& Figueroa-Lucero, G. Effects of temperature and salinity during the embryonic period of Chirostoma humboldtianum and Chirostoma riojai (Atherinopsidae) until hatching. Hidrobiológica, 23(3):365-73, 2013.

Hernández-Rubio, M. C.; Figueroa-Lucero, G.; Barriga-Sosa, I. A.; ArredondoFigueroa, J. L. \& Castro-Barrera, T. Early development of the shortfin silverside Chirostoma humboldtianum (Valenciennes, 1835) (Atheriniformes: Atherinopsidae). Aquaculture, 261:1440-6, 2006.

Ito, L. S.; Yamashita, M., Takashima, F. \& Strüssmann, C. A. Dynamics and histological characteristics of gonadal sex differentiation in pejerrey (Odontesthes bonariensis) at feminizing and masculinizing temperatures. J. Exp. Zool. A Comp. Exp. Biol., 303(6):504-14, 2005.

Kouttouki, S.; Georgakopoulou, E.; Kaspiris, P.; Divanach, P. \& Koumoundouros, G. Shape ontogeny and variation in the sharpsnout seabream, Diplodus puntazzo (Cetti 1777). Aquac. Res., 37(7):655-63, 2006.

Loy, A.; Mariani, L.; Bertelletti, M. \& Tunesi, L. Visualizing allometry: Geometric morphometrics in the study of shape changes in the early stages of the two-banded sea bream, Diplodus vulgaris (Perciformes, Sparidae). J. Morphol., 237(2):137-46, 1998.

Loy, A.; Bertelletti, M.; Costa, C.; Ferlin, L. \& Cataudella, S. Shape changes and growth trajectories in the early stages of three species of the genus Diplodus (Perciformes, Sparidae). J. Morphol., 250(1):24-33, 2001.

Macpherson, E. Ontogenetic shifts in habitat use and aggregation in juvenile sparid fishes. J. Exp. Mar. Biol. Ecol., 220(1):127-50, 1998.

Rohlf, F. J. \& Slice, D. Extensions of the procrustes method for the optimal superimposition of landmarks. Syst. Zool., 39(1):40-59, 1990.

Rohlf, F. J. On applications of geometric morphometrics to studies of ontogeny and phylogeny. Syst. Biol., 47(1):147-58, 1998.

Sheets, H. D. Software IMP () (Integrated Morphometric Package). Buffalo, Department of Physics, Canisius College, Buffalo, NY 1408, Department of Geology State University of New York at Buffalo, NY 14260, 2004.

Strüssmann, C. A.; Conover, D. O.; Somoza, G. M. \& Miranda, L. A. Implications of climate change for the reproductive capacity and survival of New World silversides (family Atherinopsidae). J. Fish Biol., 77(8):181834, 2010.

Vilchis, O. M. Modelos nulos en interacciones biológicas, una propuesta: depredación en Chirostoma riojai-zooplancton. Ciencia Ergo Sum, 7(2):1536, 2000.

Weibel, E. R.; Taylor, C. R. \& Hoppeler, H. The concept of symmorphosis: a testable hypothesis of structure-function relationship. Proc. Natl. Acad. Sci. U. S. A., 88(22):10357-61, 1991.

Youson, J. H. 2 First Metamorphosis. Fish Physiol., 11 Part B:135-96, 1988.

Zelditch, M. L.; Swiderski, D. L.; Sheets, H. D. \& Fink, W. L. Geometric Morphometrics for Biologists. A Primer. Amsterdam, Elsevier Academic Press, 2004.

\section{Dirección para correspondencia \\ Iván Alarcón-D. \\ Maestro en Biociencias \\ Doctorado en Ciencias Biológicas y de la Salud \\ UAM-I. Ciudad de México \\ MÉXICO}

E-mail: ialarcond@yahoo.com.mx

Recibido : 05-12-2016

Aceptado: $27-12-2016$ 